Research Article

\title{
An Internet of Things Evaluation Algorithm for Quality Assessment of Computer-Based Teaching
}

\author{
Jing Huang $\mathbb{B}$ \\ Department of Information and Electronic Engineering, Hunan City University, Yiyang 413000, Hunan, China \\ Correspondence should be addressed to Jing Huang; huanging@hncu.edu.cn
}

Received 17 March 2021; Revised 20 April 2021; Accepted 26 April 2021; Published 26 May 2021

Academic Editor: Muhammad Usman

Copyright $\odot 2021$ Jing Huang. This is an open access article distributed under the Creative Commons Attribution License, which permits unrestricted use, distribution, and reproduction in any medium, provided the original work is properly cited.

\begin{abstract}
Internet of Things has the ability to revolutionize computer-based teaching and assess the quality of teaching at the same time. The assessment of teaching efficiency is hampered by two issues: the evaluation index method (EIS) is insufficient, and the assessment framework is incapable of dealing with complicated fuzzy indexes. To address these issues, the theory of fuzzy stratification from IoT perspective is presented for the first time in this paper. This theory is based on the related theory of fuzzy assessment by measuring the teaching quality evaluation index. Initially, theoretical analysis and model measurement were merged to build a better multiangle EIS for teaching quality. To manage fuzzy indexes, a teaching quality assessment model was developed using both quantitative and qualitative studies. The suggested EIS and fuzzy assessment model can effectively evaluate the standard of teaching in schools, colleges, universities, and institutes, according to implementation results. This qualitative assessment approach is empirical and rational, and it strongly promotes the quality enhancement of educational effectiveness, based on our experimental and simulation results.
\end{abstract}

\section{Introduction}

Teaching evaluation is an important part of the entire teaching process. It is based on a certain teaching aspects and syllabus requirements of each subject to investigate, evaluate, and appraise teaching activities, so as to make value judgments on the quality and effect of teaching. Therefore, it is an indispensable part of the teaching process. It is an important means for in-depth education reform and improvement of teaching quality. However, the current teaching evaluation methods still remain at the level of relying on personal experience and focusing on qualitative analysis. First of all, the evaluation of teaching quality often involves multiple factors (indicators), and it must be comprehensively evaluated based on these indicators, not just from one or a few. Secondly, in comprehensive evaluation, the method of scoring each individual indicator is usually used, and then weighted average is applied. In fact, in the comprehensive evaluation problems encountered in practice, the singlefactor evaluation $[1-3]$ is often vague, and it is not appropriate to use a value absolutely. It should be represented by a fuzzy set. Therefore, in the evaluation of teaching quality, it is more appropriate to use a fuzzy comprehensive evaluation method.

Computer comprehensive evaluation is a very effective multifactor decision-making method. It is widely used in software engineering and soft science research, for example, software development cost estimation, design scheme selection, software product quality evaluation, teaching or other work quality assessments, etc. All these approaches can use the computer comprehensive assessment law [4-6].

This article will apply the relevant theories of fuzzy mathematics, combined with the multilevel indicator system of teaching quality, and propose a comprehensive computer evaluation algorithm for teaching quality. Along with that, we will discuss the role of Internet of Things in the field of education. As Internet of Things (IoT) technology has the potential to transform education at all levels, including school, college, and university, similarly, the Internet of Things (IoT) has a direct and indirect effect on learning by facilitating overall work and improving educational efficiency. In simple words, it has a broad impact on the 
teaching and learning process. That is why the evaluation area of education requires serious attention, and Internet of Things (IoT) is well suited for application in this field. Finally, the experimental results and testing of the proposed scheme are relatively analyzed. It is estimated that the planned scheme will support the teachers in the accurate teaching evaluation.

The rest of this paper is organized as follows. In Section 2 , a literature review is provided. In Section 3, we provide a comprehensive description of our proposed approach along with the application of IoT in education. In Section 4, we provide extensive simulation results. Finally, the paper is concluded and research directions for future work are provided in Section 5.

\section{Literature Review}

The use of the Internet of Things (IoT) in the educational field has provided a fantastic way to communicate and educate kids. The IoT has a major impact on the learning field. The traditional teaching practice as well as the structure of education institutions has been significantly improved with the use of IoT [7]. In the educational field, the IoT is viewed as a platform for improving an institution's infrastructure and teaching the fundamentals of computer science [8]. For an instructor or a student, be it in the classroom or on campus, IoT is equally beneficial. The effect of IoT on learning was seen in the use of sensors like Super Mechanical's Twine7 [9]. IoT allows students, instructors, and physical and virtual objects to communicate easily and efficiently [10]. The IoT is an appealing subject for learners to study the fundamentals of computer science [11]. Because of the importance of IoT, the Open University in the United Kingdom created a course called "My Digital Life" for undergraduates in the computer science department that focuses on IoT fundamentals. This program taught students how to use IoT to better understand the world and know their needs [12]. The English language will be taught using an interactive platform focused on IoT. For correcting the speech and shape of English learners' mouths, this model uses speech and visual sensors [13]. IoT is also used to teach students the fundamentals of programming. Analytical learning approaches are used to collect data and build students' learning paths. The use of the IoT as a tool to improve and simplify the educational process was comprehensively discussed in recent years. A genuine effort was made to use and apply IoT at the University of Padova [14]. Their research focused primarily on improving a web payment system for wireless communication. Their research examines the use of computing and the IoT in incorporating educational resource systems and proposes an analysis framework. Chen and Dong [15] discussed the impact of different technologies such as the IoT, cloud computing, and data mining to provide up-to-date education. Bagheri and Movahed [16] provided an overview of the educational climate as well as a distinction among intellectual and digital campuses using IoT and cloud computing. Mershad and Wakim [17] proposed an integrated model that was planned to enhance IoT in an educational world. Apart from these studies, there are many other researches in which the authors have used IoT in the educational field, as well as in other projects.

\section{Application of IoT in Education and Our Proposed Model}

The Internet of Things (IoT) appears to be changing education in terms of teaching and learning, school administration, research and education, school buildings, and so on [18]. Realistic IoT implementations in educational settings can be divided into numerous categories. For example, the data from IoT devices may be used to enhance management strategies and academic life. Learner analytics, greater access to knowledge, and personalizing learning by the use of connected devices could all be used to improve the efficacy of education and assessment. In this section, we discuss the various aspects that can benefit from the application of IoT.

3.1. Management Level. In administration, IoT may be used to simplify everyday operations that school administration would perform in order to free up further space and time for educational processes. While such IoT applications do not have a specific impact on learning, they can help prepare the school atmosphere for learning events and also save time that otherwise would have been wasted on everyday tasks. Mobile sensors, for instance, may be used to track equipment and relieve teachers of the responsibility of taking attendance and submitting to the management company on a daily basis [19]. Furthermore, IoT can endorse conventional systems engineering systems, assist in power management and conservation, control access control systems, and implement environmental monitoring and safety mechanisms for teachers and school staff [20]. Likewise, IoT devices can help track temperatures in the class room, which could have a positive impact on learning because physical surroundings temperature has been shown to influence students' awareness and behaviors [21]. In this way, the Internet of Things improves the cost-effectiveness of academic institutions. There is, however, a scarcity of research that examines the indirect effects of IoT systems on training. Rather, it is widely acknowledged that a much more relaxed and secure learning atmosphere will help students achieve better results. That is, the anticipated benefit of implementing IoT to address school administration problems. Furthermore, since these projects are typically costly to set up, many schools across the country will be unable to make predictions of funds to carry out such initiatives [22].

3.2. Learning/Teaching Level. The number of planned IoT frameworks at the instructional level is closely relevant to the construction problems, but this period at the person level. This includes the use of smart technology and other IoTbased participant tracking systems. For example, the authors of [23] proposed using a wrist tracker to gather information on learners' sleep habits and blood pressure. Because they argue, such information may be used to increase students' self-awareness of their sleeping and pressure habits, allowing 
them to determine the best time to study and relax when planning their timetable. As can be seen in these examples, IoT devices appear to have a significant effect on what academic technologists refer to as "smart schools." Many IoT initiatives, on the other hand, are focused on a specific feature of the use of IoT for academic purposes.

\subsection{Algorithm for Computer Comprehensive Evaluation.} Computer comprehensive evaluation is a complex recursive calculation process. Not only can there be any limited number of evaluation objects, but each evaluation object can contain a variety of characteristics. Therefore, computer comprehensive evaluation must take into account all aspects and repeat it to get a more ideal result.

3.3.1. Algorithm Description. The basic algorithm of computer comprehensive evaluation is as follows.

Step 1. Determine the evaluation object set $F$, factor set $U$, and comment set $V$.

Determine the object to be evaluated according to actual needs, and construct the entire object into a set, and then use fuzzy mathematical analysis to determine the set of evaluation factors and the set of comments:

$$
\begin{aligned}
\text { Object set: } F & =\left\{F_{1}, F_{2}, F_{3}, \ldots, F_{L}\right\} \\
\text { Factor set: } U & =\left\{U_{1}, U_{2}, U_{3}, \ldots, U_{n}\right\} \\
\text { Comment set: } V & =\left\{V_{1}, V_{2}, V_{3}, \ldots, V_{m}\right\}
\end{aligned}
$$

Among them, $L$ is the number of objects to be evaluated; $n$ is the number of evaluation factors; $m$ is the number of comments. In view of the fact that there are many factors that need to be considered in practical application problems, and the factors have hierarchical relationships, computer comprehensive evaluation usually adopts "multilevel comprehensive evaluation" algorithm (for the convenience of presentation, this article takes the second-level comprehensive evaluation as an example). At this time, a given factor set $U$ needs to be divided into $n$ subfactor sets $U_{1}, U_{2}, U_{3}, \ldots, U_{n}$, and the result of division should satisfy

$$
U=U_{1} \cup U_{2} \cup \cdots \cup U_{n}, U_{i} \cap U_{j}=\Phi,(i \neq j),
$$

and have

$$
U_{i}=\left\{U_{i 1}, U_{i 2}, \ldots, U_{i k_{i}}\right\}, \quad i=1,2, \ldots, n .
$$

$U_{i}$ is the first level index (composite index). Since each $U_{i}$ contains $K_{i}$ evaluation indexes, there are $\sum_{i=1}^{n} K_{i}$ in the total factor set $U$, one evaluation index, that is, the second level index (base indicator).

Step 2. Establish a factor weight distribution matrix $A_{i}(i=$ $1,2, \ldots, n)$ for each subfactor set $U_{i}(i=1,2, \ldots, n)$. Each factor in the evaluation factor set is in the "evaluation of the goal" process having different status and functions; that is, each evaluation factor occupies a different proportion in the comprehensive evaluation. This proportion is called the "priority weight value," or "weight value" for short. There are many ways to determine the "weight value"; for example, the "relative importance level calculation method" can be used.

The "weight value" determines whether it is reasonable and effective, which has a great impact on the computer comprehensive evaluation results.

Step 3. Make a single-level comprehensive evaluation of $K_{i}$ evaluation indicators in each subfactor set $U_{i}$.

For example, $U_{i}=\left\{u_{i 1}, u_{i 2}, \ldots, u_{i k_{i}}\right\}$ : the weight values of various factors are assigned as $a_{i}\left(a_{i 1}, a_{i 2}, \ldots, a_{i k_{i}}\right)$, where $a_{i j}>0$, and the comment set of $U_{i}$ is $V_{i}=\left\{v_{i 1}, v_{i 2}, \ldots, v_{i k_{i}}\right\}$. Carrying out a single-factor evaluation on each factor of $U_{i}$, the evaluation matrix $S_{i}$ can be obtained, which is,

$$
S_{i}=\left[\begin{array}{c}
s_{i 1} \\
s_{i 2} \\
\vdots \\
s_{i k_{i}}
\end{array}\right] .
$$

Among them, $s_{i 1}, s_{i 2}, \ldots, s_{i k_{i}}$ represent the score of each factor evaluation object.

The so-called single-level comprehensive evaluation of the subfactor set $U_{i}$ is actually to calculate the single-factor comprehensive evaluation matrix $R_{i}(i=1,2, \ldots, n)$.

$$
R_{i}=A_{i} \cdot S_{i} \text {. }
$$

This means after implementing $S_{i}$ transformation on the input matrix (array) $A_{i}$, the output matrix (array) $R_{i}$ can be obtained. Obviously, when $A_{i}$ and $S_{i}$ are known, compound operations can be performed:

$$
R_{i}=A_{i} \cdot S_{i}=\{r\} .
$$

Theoretically speaking, the above compound expressions have infinitely many kinds of calculation models. However, in the actual application process, the "weighted average" comprehensive evaluation calculation model is the most effective, because it balances all the evaluation factors according to the "weight value," which are applicable to situations where overall indicators are required.

In the "weighted average" comprehensive evaluation operation model, the weighted average operator is denoted as $M(\bullet,+)$. Here, "๑" means ordinary real number multiplication and "ten" means ordinary real number addition.

Step 4. After comprehensively evaluating the $n$ factors $U_{i}$ of $U$, the total comprehensive evaluation matrix $R$ is obtained:

$$
R=\left\{\begin{array}{c}
R_{1} \\
R_{2} \\
\vdots \\
R_{n}
\end{array}\right\} .
$$

Step 5. Perform matrix compound operation to calculate the comprehensive evaluation result (comprehensive evaluation value) $B$ of the object to be evaluated. 
Suppose the weight value distribution matrix of $U$ is $A$. Then $B=A \times R$. The operator is the same as above. It is not only the result of $U$ 's secondary computer comprehensive evaluation, but also the comprehensive evaluation result of all factors of $U$. That is:

$$
B=A \cdot R=A\left[\begin{array}{c}
R_{1} \\
R_{2} \\
\vdots \\
R_{n}
\end{array}\right]=A \cdot\left[\begin{array}{c}
A_{1} \circ S_{1} \\
A_{2} \circ S_{2} \\
\vdots \\
A_{n} \circ S_{n}
\end{array}\right] .
$$

According to the abovementioned two-level computer comprehensive evaluation principle (Steps 2-5), the evaluation of $L$ objects to be evaluated can be successfully completed and finally the comprehensive evaluation values of $L$ different objects to be evaluated, namely $B_{1}, B_{2}, \ldots, B_{L}$.

Step 6. Sort the $L$ comprehensive evaluation values $B_{i}(i=$ $1,2, \ldots, L) 21$ and output the sorting result.

At this point, the entire computer comprehensive evaluation work is announced. Obviously, for teaching quality evaluation, the larger the evaluation value, the higher the teaching quality level, and the smaller the evaluation value, the lower the teaching quality level.

3.3.2. Block Diagram of Algorithm Programming. In order to facilitate computer implementation, the following is a flowchart (simplified diagram) of the computer comprehensive evaluation algorithm. This flowchart of Figure 1 is based on the basic computer comprehensive evaluation algorithm.

Users can choose any program design according to their needs. Language compiles computer comprehensive evaluation program.

\subsubsection{Computer Comprehensive Evaluation Index System for} Teaching Quality. Teaching quality is a fuzzy concept. When people form a concept in their minds, it has a certain connotation and extension. The entire set of objects that conform to this concept is the extension of this concept. For teaching quality, it is both there is no clear extension and the connotation is quite complicated. For its evaluation, generally only a few representative indicators can be selected for assessment.

Considering that the teaching process is complex composed of many factors, the quality of teaching is often reflected from different aspects. Therefore, when evaluating teaching, it is necessary to carry out many aspects of the teaching process from different perspectives and different aspects. It is necessary to evaluate the final teaching effect, but also pay attention to the role played by each link in the teaching process and treat them as a whole. When applying fuzzy mathematics to analyze practical problems, the first step is to establish a hierarchy in the substructure diagram, the highest level is the general goal, the middle level is the criteria to be followed when making decision analysis, and the lowest level is the evaluation index level. Generally

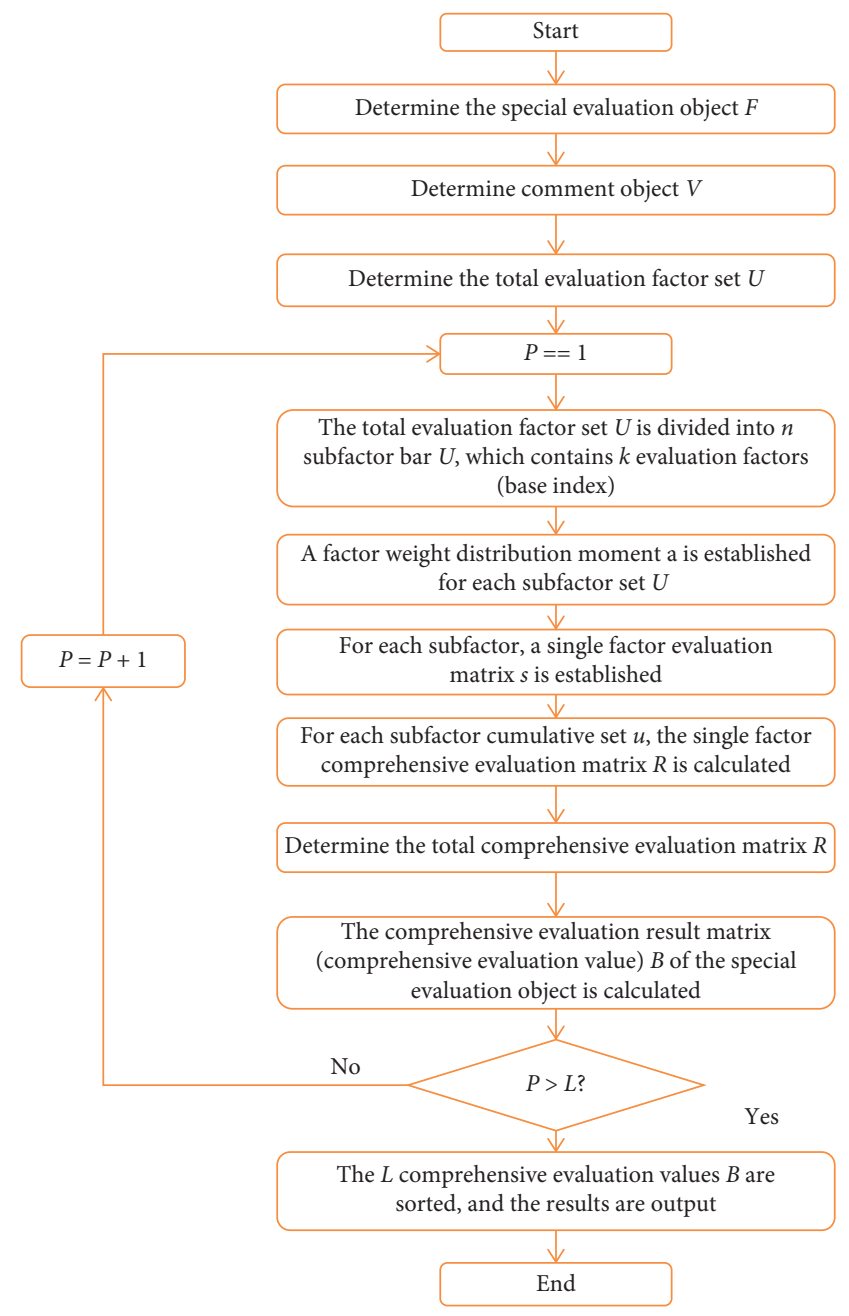

FIGURE 1: Flow chart of computer comprehensive evaluation (level 2).

speaking, the indicators reflecting the quality of teaching can be summarized into the following 5 aspects:

(1) Teacher preparation: this indicator reflects the overall level of teacher preparation, such as teaching purpose, teaching preparation, and homework design.

(2) Textbook processing: this indicator reflects the ability of teachers to control textbooks and teach students in accordance with their aptitude. Such as the scientific nature of knowledge transfer, the processing of key and difficult points, and the cultivation of student abilities.

(3) Implementation of teaching methods: it reflects whether the structure and arrangement of classroom teaching are reasonable, whether the selection and combination of teaching methods are appropriate, and whether the cooperation between teachers and students is tacitly compatible, such as the overall design of the classroom, teaching bilateral activities, the combination of teaching methods, and the use of teaching methods. 
(4) Teaching quality: it reflects the degree of influence of teachers' teaching quality on teaching quality, including teaching attitude, teaching language, blackboard writing, and demonstration.

(5) Teaching effect: it reflects the final result of the teaching process, including the classroom teaching atmosphere, the situation of completing the teaching plan, the situation of students mastering knowledge, and the improvement of students' thinking ability.

The above five types of indicators constitute an indicator system that reflects the level of teaching quality, which can be expressed as a hierarchical analysis diagram. In actual application, the indicators should be independent and inclusive of each other.

3.3.4. Determination of the Weight Value of the Comprehensive Evaluation Index of Teaching Quality. The comprehensive evaluation indicator system has multiple indicators and multiple levels, and each indicator has different effects on the indicators of the previous layer. In actual comprehensive evaluation, different weight values are often used to indicate the size of its effect. But the determination of the weight value is often subjective factors. In order to make the weight value of each indicator reflect the objective reality as much as possible, the method of $0 \sim 4$ score in Table 1 is used to determine the relative importance weight of these indicators.

The specific steps are as follows.

(1) Draw up an expert score sheet and ask each expert to fill it out separately.

Scoring method: compare $n$ evaluation indicators in pairs; the very important side scores 4 points, the less important side scores 0 points, the more important side scores 3 points, the less important side scores 1 point, and the two equally important sides score 2 points. The total score of $0 \sim 4$ is $\sum_{i=1}^{n} C_{i}=2 n(n-1)$, where $n$ is the number of indicators.

(2) There are $m$ experts to participate in the scoring, and the $j^{\text {th }}$ expert's scoring value for the $i^{\text {th }}$ index is $C_{i j}(i=1,2, \ldots, m)$ calculating the score $Z$ of each index,

$$
Z_{i}=\sum_{j=1}^{m} C_{i j}, \quad i=1,2 \ldots, n
$$

(3) Calculate the total score

$$
Z=2 m m(n-1) \text {. }
$$

(4) Calculate the weight value of each indicator

$$
A_{i}=\frac{z_{i}}{z}, \quad i=1,2 \ldots, n \text {. }
$$

According to the above method, fill in the form through

\begin{tabular}{|c|c|c|c|}
\hline Index & $X_{1}, X_{2}, \ldots, X_{n}$ & Score & Proportion \\
\hline $\begin{array}{l}X_{1} \\
X_{2}\end{array}$ & & $\begin{array}{l}C_{1} \\
C_{2}\end{array}$ & $\begin{array}{l}C_{1} / \sum C_{1} \\
C_{1} / \sum C_{1}\end{array}$ \\
\hline $\begin{array}{l}X_{n} \\
\sum\end{array}$ & & $\begin{array}{c}\ddot{C}_{n} \\
\sum C_{i}\end{array}$ & $\begin{array}{c}C_{n} / \sum C_{n} \\
1.000\end{array}$ \\
\hline
\end{tabular}
the scores of the experts, and calculate the importance
TABLE 1: 0 4 score table.

weight of each level index relative to the upper level index as shown in Table 2.

3.3.5. Mathematical Model of Computer Comprehensive Evaluation of Teaching Quality. Suppose the object set to be evaluated is $F=\left\{F_{1}, F_{2}, F_{3}, \ldots, F_{L}\right\}$, the comment set is $V=$ $[0,1]$, and a continuous real number interval is acceptable. Hence, the following needs to be considered.

(1) Determine the Score Value of Each Base Indicator. The scoring value of each base indicator is scored by the members of the teaching quality assessment team on the 17 base indicators on a percentile system, and these scoring values are relatively independent.

(2) Evaluation of Base Indicators. The evaluation of the base index is to determine the membership function $\mu s\left(X_{i}\right)$ of each base index $X$ to the comment set $V$ and find its evaluation value $s_{j}$. According to the theory of fuzzy mathematics, the membership function can be defined as the following evaluation model:

$$
s_{i}=\mu_{s}\left(X_{i}\right)=\left\{\begin{array}{l}
0\left(0 \leq X_{i}<50\right), \\
\frac{1}{50} X_{i}-1\left(50 \leq X_{i} \leq 100\right) .
\end{array}\right.
$$

3.3.6. Evaluation of Composite Indicators. The evaluation of the composite index is the multilevel computer comprehensive evaluation. Let $U$ be the ith $(i=1,2, \ldots, 5)$ evaluation subset in the overall goal of teaching quality, and the corresponding importance weight vector is $A$, and the membership degree (evaluation value) vector that has been obtained from the evaluation of the base index is $S$. Use the fuzzy relation composition operation (the operator is $M(\mathbf{e}$, $+)$ ) to obtain the evaluation value of the composite index for the overall goal of teaching quality for

$$
R_{i}=A_{i} \cdot S_{i}=\sum_{i=1}^{k} a_{i j} S_{i j}
$$

where $a_{i j} \in[0,1]$ and $\sum_{i=1}^{k} a_{i j}=1, s_{i j} \in[0,1], k=3$ or 4 .

3.3.7. Comprehensive Evaluation of the General Objective. The comprehensive evaluation of the overall goal is a multilevel and two-level computer comprehensive evaluation, which is a comprehensive evaluation based on a composite index evaluation. 
TABLE 2: The importance weight for each level index relative to the upper level index.

\begin{tabular}{|c|c|c|c|}
\hline Index & Weight & Index & Weight \\
\hline$F_{1}$ : teachers prepare lessons & 0.19 & $X_{7}:$ overall classroom design & 0.27 \\
\hline$F_{2}:$ textbook processing & 0.16 & $X_{8}$ : bilateral teaching activities & 0.22 \\
\hline$F_{3}$ : teaching method implementation & 0.20 & $X_{9}$ : teaching methods & 0.26 \\
\hline$F_{4}$ : teaching quality & 0.19 & $X_{10}$ : application of teaching means & 0.25 \\
\hline$F_{5}$ : teaching effect & 0.26 & $\sum$ & 1.00 \\
\hline$\sum$ & 1.00 & $X_{11}$ : teaching attitude & 0.38 \\
\hline$X_{1}$ : teaching purpose & 0.35 & $X_{12}$ : teaching language & 0.32 \\
\hline$X_{2}$ : teaching preparation & 0.41 & $X_{13}$ : blackboard writing and demonstration & 0.30 \\
\hline$X_{3}$ : job design & 0.24 & $\sum$ & 1.00 \\
\hline$\sum$ & 1.00 & $X_{14}:$ classroom atmosphere & 0.20 \\
\hline$X_{4}:$ the science of knowledge transfer & 0.37 & $X_{15}$ : completion of teaching plan & 0.23 \\
\hline$X_{5}:$ treatment of key and difficult points & 0.32 & $X_{16}$ : students' knowledge & 0.27 \\
\hline$X_{6}:$ the cultivation of students' ability & 0.31 & $X_{17}$ : the improvement of students' thinking ability & 0.30 \\
\hline$\sum$ & 1.00 & $\sum$ & 1.00 \\
\hline
\end{tabular}

$$
B=A \cdot R=\sum_{i=1}^{5} A_{i} R_{i} .
$$

$B \in[0,1]$ is the final evaluation value.

Through the above Steps $1-4$, the relative evaluation results of the $L$ objects to be evaluated $F, F_{z}, F_{L}$ to measure the teaching quality can be obtained $Y=B_{1}, B_{2}, \ldots, B_{L}$, where $B \in[0,1]$ and the value of $B$ reflects the level of teaching quality. The larger the value, the higher the teaching quality. According to this, $F_{1}, F_{2}, \ldots, F_{m}$ represents the order of teaching quality.

3.3.8. Application Examples. As for the application of the abovementioned comprehensive computer evaluation algorithm for teaching quality, four teachers from different teaching and research sections of a technical secondary school are selected as the evaluation objects. According to the evaluation index system of teaching quality, from teacher preparation, textbook processing, teaching method implementation, teaching quality, and teaching effect 5 subobjectives, we comprehensively evaluate the teaching quality of 4 teachers: $A, B, C$, and $D$ and sort them by the size of the evaluation value. The scoring of the base index is the basis of the entire evaluation process. In order to make the scoring value of the base index true, it reflects the actual teaching situation of teachers. For different basic indicators, different methods are used to score the following.

(1) Test Method. Use different forms such as written examination, oral examination, and practical operation to assess students' mastery, understanding, and application of knowledge.

(2) Observation Method. The members of the evaluation team are immersive and obtain first-hand information of teaching activities in a natural state and then make a score. It is mainly used to score basic indicators such as teachers' teaching attitude, teaching language, classroom teaching atmosphere, and teaching bilateral activities.

(3) Investigation Method. These methods include holding a symposium, filling out a written survey form, interviewing students and parties, understanding the situation, and making an assessment.

(4) Inspection Method. Check the teacher's lesson preparation notes, homework correction records, student work, etc., to understand the information to be mastered when grading. After completing the above preparations, you can start the evaluation.

3.3.9. Base Index Evaluation. The members of the teaching quality evaluation team scored the 4 teachers one by one according to 17 base indicators and then calculated the average score for each base indicator as the score value of the base indicator. Score one by one, and calculate the average score for each base indicator as the score value of the base indicator. Substitute the score value into the membership function to calculate the degree of membership, which is the evaluation result of the base index.

3.3.10. Evaluation of Composite Indicators. According to the importance weight of the base index and the composite index evaluation mathematical model, the evaluation results of all composite indicators can be calculated. For example, the calculation of the composite indicator $\mathrm{Ui}$ of teacher $A$ in Table 3:

$$
R_{1}=(0.35,0.41,0.24) \cdot\left[\begin{array}{l}
0.76 \\
0.68 \\
0.82
\end{array}\right]=0.35 \cdot 0.76+0.41
$$

In the same way, all the composite index evaluation values of each teacher can be calculated, as shown in Table 3.

\subsubsection{Comprehensive Evaluation of the Overall Goal.} According to the composite index importance weight and the overall objective comprehensive evaluation mathematical model, the comprehensive evaluation results of the four teachers' teaching quality are calculated (as shown in Table 4). 
TABLE 3: Evaluation results of composite indicators.

\begin{tabular}{|c|c|c|c|c|c|}
\hline Composite index & Evaluation value & JIA & YI & BING & DING \\
\hline$U_{1}$ & $R_{1}$ & 0.7416 & 0.7074 & 0.6736 & 0.8266 \\
\hline$U_{2}$ & $R_{2}$ & 0.5272 & 0.4208 & 0.5230 & 0.5672 \\
\hline$U_{3}$ & $R_{3}$ & 0.6150 & 0.6006 & 0.6660 & 0.6500 \\
\hline$U_{4}$ & $R_{4}$ & 0.5700 & 0.4656 & 0.6528 & 0.5928 \\
\hline$U_{5}$ & $R_{5}$ & 0.6526 & 0.6246 & 0.5782 & 0.6188 \\
\hline
\end{tabular}

TABLE 4: Comprehensive evaluation results.

\begin{tabular}{lccrr}
\hline Teacher & JIA & YI & BING & DING \\
\hline Comprehensive evaluation value $b$ & 0.6263 & 0.5727 & 0.6192 & 0.6513 \\
\hline
\end{tabular}

For example, we have the overall goal $U$ for teacher $A$ and comprehensive evaluation value calculation.

$$
B=(0.19,0.16,0.20,0.19,0.26) \cdot\left[\begin{array}{l}
0.7416 \\
0.5272 \\
0.6510 \\
0.6526
\end{array}\right]=0.19 \cdot 0.7416+0.16 \cdot 0.5272+0.20 \cdot 0.6510+0.26 \cdot 0.6526=0.6263
$$

In the same way, the comprehensive evaluation value $B$ of other teachers can be obtained. It can be seen that the order of the four teachers' teaching quality level from high to low is $D, A, C, B$.

From the final evaluation results, it can better reflect the actual teaching quality level of the four teachers, achieve the expected evaluation goals, and achieve satisfactory results.

3.3.12. Fuzzy Logic Assessment. We proposed an infrastructure that enables a first evaluation of the learners' approach $[24,25]$ to solve the first challenges they may face, when designing their solution. Without any of the involvement of a teacher, students will ask the system what is incorrect with the answer they are creating. Our goal is to build a framework that aids learners in comprehending what they are learning. There are several methods to assess computer learning practices, where [26] has a strong survey on static or dynamic evaluation of computer software as shown in Figure 2.

As seen in the diagram above, the instructor first asks for an integration of the optimal estimated algorithm for solving a problem. Following that, a number of software parameters that influence its architecture will be determined. As a result, we have an example of the ideal estimated algorithm. Then, by each parameter, fuzzy numbers will be generated in the following way: each fuzzy set will begin as a default triangular function centered on the estimated algorithm's metric value; the teacher may easily change the fuzzy set suggesting the following:

(i) The solution's highest values that the educator finds low

(ii) The bare min of validity

(iii) The highest possible score for accuracy (iv) The solution's min value that the educator finds high

\section{Experimental Results}

4.1. Experiment. The experimental results of our proposed system can be divided into the following stages.

4.1.1. Choice of Teaching Quality Analysis Cases. This paper will conduct performance testing of related models after choosing the model method and design. Developers of network teaching methods have increasingly developed many common course models for various educational needs in recent times. Traditional personal mentoring programmes, student-centered personal mentoring classes, personalized online education classes, knowledge-first personal mentoring classes, explorative personal mentoring classes, and conceptual personal mentoring classes are some of the new trajectory structures available. This article suggests an Internet of Things evaluation algorithm for Computer Teaching Quality Model which is a combination of wireless sensor networks and fuzzy comprehensive evaluation algorithms to address the aforementioned issues. On this foundation, we will choose teaching quality diagnostic situations for performance monitoring based on relevant regulations.

4.1.2. Test for Teaching Quality Diagnostic Performance. As compared to other models, the design has obtained strong experimental performance, as seen in Figure 3. The failure rate of network detection of teaching quality diagnosis reduced as the number of test samples increased, and much more accurate results have been obtained. The efficiency of the convolutional neural network is quite high as 


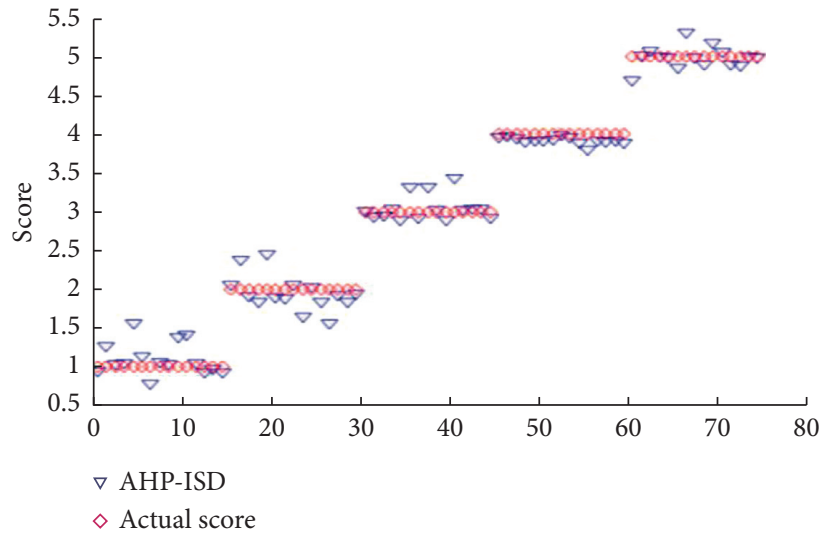

Figure 2: Fuzzy logic.

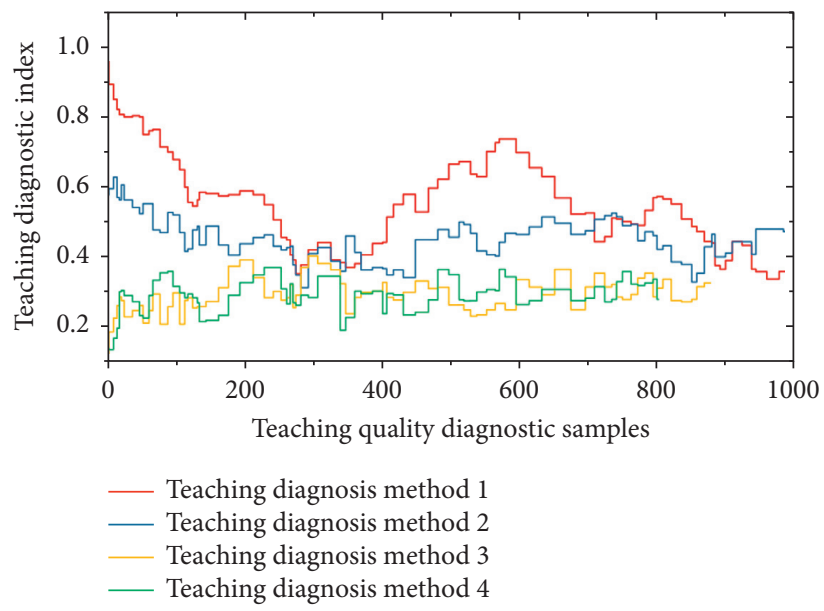

FIGURE 3: Judgment achievement rate of the teaching quality diagnosis system.

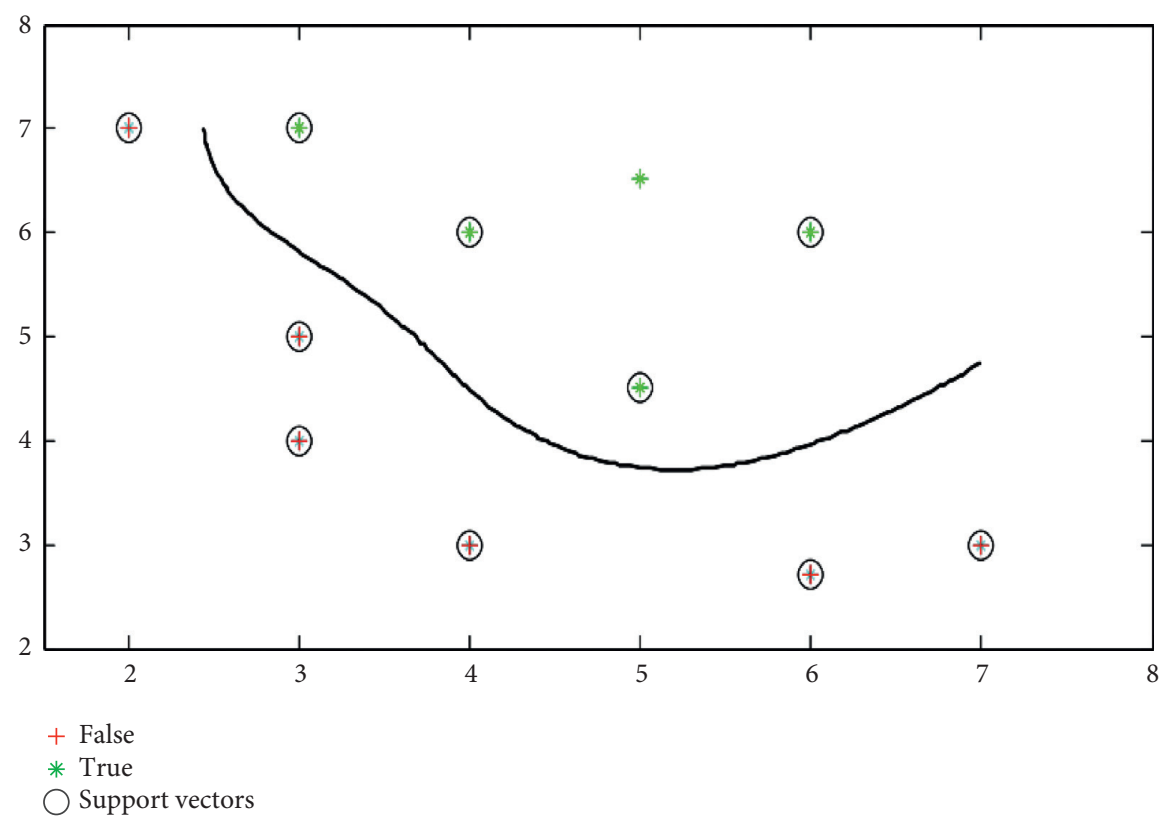

FIGURE 4: SVM exercise outcomes when $\lambda=0.6$ evaluation result. 
compared to many other algorithms, as per the results. As a result, the test results reveal that convolutional neural networks' extraction performance is significantly higher than other approaches' data mining capacities and that they can give better effective information mining and retrieval for the teaching quality diagnosis process.

4.2. Teaching Quality Evaluation (TQE) of Support Vector Machine (SVM) Grounded on Analytic Hierarchy ProcessASD. The following is the SVM teaching quality assessment method focused on AHP-ISD:

Stage 1. The data from each assessment is normalized in this process, and a teaching quality decision matrix is developed

Stage 2. During this stage, the weight of AHP as well as ISD is designed

Stage 3. During this stage, the comprehensive weight of AHP-ISD is designed

Stage 4. During this stage, the development directory data is separated into two sets such as test set and trained set

Stage 5. Here the SVM prediction model is set up by our trained data set

Stage 6. This is the last stage, where the SVM teaching quality evaluation design is tested

The evaluation result of SVM exercise outcomes when $\lambda=0.6$ can be explained in Figure 4 .

\section{Conclusion}

This paper applies the related theories of fuzzy mathematics and uses the analytic hierarchy process to put forward a new idea of comprehensive evaluation of teaching quality by computer, so as to make a preliminary exploration of the transformation of teaching quality evaluation from qualitative analysis to quantitative analysis. We have addressed different issues of teaching quality and designed a model to solve these issues. We have the theoretical analysis and model measurement to build a better multiangle EIS for teaching quality. To manage fuzzy indexes, a teaching quality assessment model was developed using both quantitative and qualitative studies. Practical results show that the use of teaching quality computer synthesis as the evaluation method can efficiently achieve the expected evaluation goal and obtain satisfactory results. For future we have planned to increase the efficiency of teaching quality evaluation. Furthermore, we have planned to use different AI techniques to improve the teaching quality and to create smart teacher technique which will increase teaching quality.

\section{Data Availability}

The data used to support the findings of this study are included within the article.

\section{Conflicts of Interest}

The author declares that there are no conflicts of interest.

\section{References}

[1] M. Zhao and W. Zhan, "Teaching quality evaluation system based on deep learning algorithm," Modern Electronic Technology, vol. 43, no. 13, pp. 143-146, 2020.

[2] C. Yu, "Research on innovative teaching of basic courses of computer application in the big data era," Talent, vol. 12, no. 18, p. 135, 2020.

[3] H. Jin, Z. Li, M. Qi, and F. Jian, "Research on comprehensive ability evaluation model of computer professionals," Software Guide, vol. 19, no. 6, pp. 257-260, 2020.

[4] Li Pei, "Research on the evaluation index system of computer network technology practice teaching quality based on big data," Network Security Technology and Application, vol. 5, pp. 99-100, 2020.

[5] X. Zhong and R. Li, "Research on the process control of student evaluation on teaching quality of university teachers," Education Review, vol. 2, pp. 61-65, 2020.

[6] Q. Yang, Y. Lian, T. Zhao, and H. Yuan, "Research on the improvement of teaching quality of computer mathematics courses," Education Modernization, vol. 7, no. 17, pp. 51-52, 2020.

[7] M. Veeramanickam and M. Mohanapriya, "IOT enabled futurus smart campus with effective E-learning: i-campus," GSTF Journal of Engineering Technology (JET), vol. 3, no. 4, p. 81, 2016.

[8] H. F. Elyamany and A. H. AlKhairi, "IoT-academia architecture: a profound approach," in Proceedings of the 2015 16th IEEE/ACIS International Conference on Software Engineering, Artificial Intelligence, Networking and Parallel/Distributed Computing (SNPD), June 2015.

[9] S. Gul, M. Asif, S. Ahmad, M. Yasir, M. Majid, and M. Arshad, "A survey on role of internet of things in education," IJCSNS, vol. 17, no. 5, pp. 159-165, 2017.

[10] J. Marquez, J. Villanueva, Z. Solarte, and A. Garcia, "IoT in education: integration of objects with virtual academic communities," in Proceedings of the 2016 World Conference on New Advances in Information Systems and Technologies, Recife, Brazil, March 2016.

[11] J. Chin and V. Callaghan, "Educational living labs: a novel internet-of-things based approach to teaching and research," in Proceedings of the 2013 9th International Conference on Intelligent Environments (IE), July 2013.

[12] Y. Wang, "English interactive teaching model which based upon internet of things," in Proceedings of the 2010 International Conference on Computer Application and System Modeling (ICCASM), October 2010.

[13] H.-C. Cheng and W.-W. Liao, "Establishing an lifelong learning environment using IOT and learning analytics," in Proceedings of the 2012 14th International Conference on Advanced Communication Technology (ICACT), IEEE, PyeongChang, South Korea, February 2012.

[14] A. P. Castellani, N. Bui, P. Casari, M. Rossi, Z. Shelby, and M. Zorzi, "Architecture and protocols for the internet of things: a case study," in Proceedings of the 2010 8th IEEE International Conference on Pervasive Computing and Communications Workshops (PERCOM Workshops), March 2010.

[15] Y. Chen and X. Dong, "The development and prospect of new technology in modern distance education," in Proceedings of the 2013 International Conference on Information Science and 
Computer Applications (ISCA 2013), Tel Aviv, Israel, October 2013.

[16] M. Bagheri and S. H. Movahed, "The effect of the internet of things (IoT) on education business model," in in Proceedings of the 2016 12th International Conference on Signal-Image Technology and Internet-Based Systems (SITIS), November 2016.

[17] K. Mershad and P. Wakim, "A learning management system enhanced with internet of things applications," Journal of Education and Learning, vol. 7, no. 3, p. 23, 2018.

[18] Z. Tianbo, "The Internet of things promoting higher education revolution," in Proceedings of the Fourth International Conference on, Multimedia Information Networking and Security (MINES), pp. 790-793, IEEE, Nanjing, China, November 2012.

[19] B. D. Ralhan, " 6 ways the internet of things is transforming the education sector: Featurephilia," 2017, http://indiatoday. intoday.in/education/story/internet-ofthings-transformingeducation/1/870592.html.

[20] C. Benson, "The Internet of things, IoT systems, and higher education," Educause Review, vol. 51, pp. 34-43, 2016.

[21] S. Mishra, "12 modern learning practices with internet of things," 2017, http://www.clickonf5.org/internet/modernlearning-practices-iot-education/126745.

[22] I. Asseo, M. Johnson, B. Nilsson, C. Neti, and T. J. Costello, "The internet of things: riding the wave in higher education," Educause Review, vol. 51, pp. 11-33, 2016.

[23] F. De Arriba-Pérez, M. Caeiro-Rodríguez, and J. M. SantosGago, "Towards the use of commercial wrist wearables in education," in Proceedings of the 2017 4th Experiment@International Conference (exp. at'17), June 2017.

[24] M. Ben-Ari, "Constructivism in com-puter science education," Journal of Computers in Mathematics and Science Teaching, vol. 20, no. 1, pp. 45-73, 2001

[25] D. Traynor, S. Bergin, and J. P. Gibson, "Automated assessment in CS1,"vol. 52, pp. 223-228, in Proceedings of the 8th Australian Conference on Computing Education, vol. 52, pp. 223-228, Australian Computer Society, Inc., Darlinghurst, Australia, January 2006.

[26] K. M. Ala-Mutka, "A survey of automated assessment approaches for programming assignments," Computer Science Education, vol. 15, no. 2, pp. 83-102, 2005. 